\title{
Beyond our labour pains: women experiences of informal payments and delivery room conditions at birth in the Bongo district of Ghana
}

\author{
Martin Amogre Ayanore ${ }^{1 *}$, Robert Kuganab-lem², Paul Armah Aryee ${ }^{3}$ \\ ${ }^{1}$ Department of Health Services Research, CAPHRI, Maastricht University, Maastricht, Netherlands \\ ${ }^{2}$ School of Allied Health Sciences (SAHS), University for Development Studies, Tamale, Ghana \\ ${ }^{3}$ Department of Community Nutrition, School of Allied Health Sciences (SAHS), University for Development Studies, \\ Tamale, Ghana
}

Received: 09 May 2016

Accepted: 02 June 2016

*Correspondence:

Dr. Martin Amogre Ayanore,

E-mail: m.ayanore@maastrichtuniversity.nl

Copyright: (c) the author(s), publisher and licensee Medip Academy. This is an open-access article distributed under the terms of the Creative Commons Attribution Non-Commercial License, which permits unrestricted non-commercial use, distribution, and reproduction in any medium, provided the original work is properly cited.

\begin{abstract}
Background: Evidence of poor delivery practices is rife in northern Ghana. The study aims to unearth women perceptions of delivery and informal payments conditions in rural Ghana.

Methods: Quantitative and qualitative data was collected between March-May 2015 in study district. A survey questionnaire collected data among 90 eligible women whiles FGD $(n=17)$ and IDI $(n=15)$ data was collected among different groups of women.

Results: Women who ever paid informally ( $\mathrm{aOR}=11.3,95 \%$ CI. $0.637-200.428$ ) were more willing to pay informally for accessing maternity care. Women expression of informal payments as inevitable (aOR $=4.3$, 95\% CI 0.056 326.999) influenced their willingness to pay informally. Expression of informal payments as gratitude was 5 times likely to influence willingness to pay informally.

Conclusions: To meet women expectation for clean, friendlier and supportive delivery services, facility environments must create conducive space for women use of delivery services. Identifying mechanisms to improve delivery care conditions is important to promote good maternity standards.
\end{abstract}

Keywords: Delivery room conditions, Informal payments, Rural Ghana

\section{INTRODUCTION}

After the Nairobi safe motherhood conference in 1986, stakeholder consensus and support has brought progress for advancing women health issues globally. ${ }^{1}$ Notable progress in antenatal and skilled delivery care improved between 1990 and 2003 (from 41\% to 57\%) among many countries in the developing world. ${ }^{2}$ In developing countries where access to delivery care has improved, poor quality and barriers for facility based utilization are often evident. ${ }^{3}$ Women perceptions of poor delivery care impacts negatively on birth service utilization in some settings. The evidence of women shying away from birthing services at the facility due to social and health system barriers are documented. ${ }^{4-6}$ Furthermore, women acceptance and utilization of facility based birthing services is often characterized by their perceptions of the environmental conditions where these services are provided. One study showed health-provider relational attitudes and clinic environments as important in determining women delivering service utilization levels. ${ }^{7}$

In northern Ghana, evidence posits poor delivery practices exist. ${ }^{8,9}$ Evidences from other context show and the removal of these perceived poor delivery conditions for women could help improve women facility based birthing outcomes. ${ }^{10}$ Empirical evidence existing in northern Ghana recounts patient inequalities, logistical 
and resource constraints as militating factors for good maternity service delivery outcomes. ${ }^{11-14}$ Additionally, informal payments in Ghana are attributed to poor health funding gaps and poor conditions of service for providers, typical of many developing countries. In the Upper East Region of Ghana only $35 \%$ of deliveries recorded in 2008 were supervised by qualified midwives, despite national and regional health efforts for greater skilled utilization. ${ }^{15-17}$ Informal payments within the health care system has also been evidenced to impact on service utilization in Ghana. ${ }^{18,19}$ Moreover, women have strong perceptions of delivery care omissions and commissions within the health system.

Studies investigating women's accounts of interactions in delivery care have been documented in Ghana, however, no study investigated both women's perspectives of facility delivery and informal payment conditions for maternity care. ${ }^{9,20-23}$ This study aims to fill this knowledge gap in Ghana by examining women perceptions of facility delivery and informal payments experiences in rural Ghana. Examining these two measures; facility delivery conditions and informal payments provide a window of opportunity to unearth salient underpinnings affecting women use of birthing services in rural Ghana. Applying a mixed study approach in a homogenous setting in Ghana, the findings will inform policy and service delivery activities to improve current facility based delivery services across Ghana.

\section{METHODS}

\section{Study design and setting}

The study adopted a descriptive cross-sectional approach where both qualitative and quantitative data was collected. Quantitative data was collected using a semistructured interviewer questionnaire. Based on the themes of the questionnaire, interview guides were developed to collect qualitative data using in-depth interviews (IDIs) and Focus group discussions (FGDs). A mix of quantitative and qualitative techniques was applied to provide in depth saturation of data for the two main study outcomes; delivery conditions and informal payment experiences. Since these two issues are considered sensitive and difficult to assess within the study setting, it was appropriate to ensure a triangulation of available evidence on the study aim. Adopting a mixed method approach enabled participants provide interactive responses and insights into survey data findings. The first arm of the study regarding the questionnaire contained questions that sought to investigate participant's perceptions regarding informal payments for maternity care. The second arm assessed participant's perceptions of delivery room conditions during their last births. The study was conducted in three communities in the Bongo district of northern Ghana. Typical of any rural district in Ghana, subsistence agriculture is the main source of livelihoods across the district. At the time of the study, the district had one hospital, five primary healthcare centres and thirty five community-based health planning and services (CHPS) compounds. CHPS compound concept is the lowest level of primary health care available predominantly across northern Ghana.

\section{Study participants and sampling}

We recruited women aged 18-35, comprising of pregnant women and women with recent child births prior to the study. We used a convenient sampling approach which allowed us to recruit only eligible respondents (pregnant and recent births $<24$ months) at the community and health facility level. One sub-district (Namoo) out of a total of six health sub-districts was purposively selected for the study. Eligible respondents were recruited in two phases; firstly, three randomly selected communities; Namoo, Boko, and Amanga were selected from the Namoo sub-district health register. In each community, 30 eligible women were purposively recruited as respondents for the quantitative arm of the study. Secondly, purposive sampling selected 5 women from each community level (total of 15) during the administration of the questionnaire to provide further indepth interviews (IDIs). Women who showed intense interest and were actively involved in their communities activities as leaders were selected. Two 2 FGDs were held: one focus discussion in Amanga community $(n=7)$ whiles another was held in Namoo health centre $(n=10)$. Table 1 provides a summary of participants and key themes explored for both quantitative and qualitative measures.

\section{Outcome measures}

Two outcome measures were assessed in the study; facility based delivery conditions and informal payments for delivery and post-delivery care. Facility based delivery here referred to women who gave birth at a health facility (private/public) at their last birth. As outlined by Koblinsky, facility based delivery conditions constitutes all other enabling factors such as issues on community setting attributes, state of clinic and its arrangements for delivery care, relational attributes, and logistics for service provision. Delivery conditions are known to impact women perceived acceptance of delivering within a facility level. ${ }^{5,23-26}$ Based on these broad concepts of facility based delivery, this study explicitly examined women's perceptions of staff attitudes during delivery sessions, delivery room floor conditions and linings, privacy at delivery rooms, and travel distance/time to facility. Others included hospital arrangements and birth preparedness plans, and intentions for future delivery in a health facility. Intentions for future delivery were included to serve as a proxy measure to examine women's future intentions for facility delivery after their previous experiences with delivery conditions. Informal payment in this study refers to cash and gift payments made by women or relative to delivery care, postnatal and postpartum family planning visits to the 
health facility. It was important to assess this measure relative to delivery care to unearth existing inequity issues affecting women meeting essential maternal care needs. Other studies in extending this definition define informal payments as cash or in-kind payments that may happen alongside formal official payment for health care services. $^{27,28}$ These payments when suspected to violate accountability mechanisms within the health system may create inefficiencies and inequity issues with the use of equal services by all patients. ${ }^{27,29,30}$

\section{Data collection}

Quantitative and qualitative data were collected sequentially between March-May 2015. The survey component consisted of 20 questions informed by existing literature on the study aim. Questionnaire questions were in three parts; Part 1 explored women ever experiences of informal payments during delivery and general health care seeking. Part 2 and 3 explored women perceptions and willingness to pay informally for delivery care and post-delivery maternity care services. To recruit eligible women participants in each community level to respond to the questionnaire, all 3 selected communities were visited and meetings with community leaders were held to seek approval to engage women in the study. This was important considering the social setting for the study where men's approval was important in gaining support for such an activity. An initial census was undertaken in each community level and sampled households visited to recruit eligible participants. After the initial census, eligible women with varied populations of and for Boko, Amanga and Namoo communities respectively were recorded. ${ }^{32,38,62}$ To ensure homogeneity across all study communities and meet available resources for data collection, an average quota of 30 women in each community were purposively selected as eligible survey respondents. Where a household had more than one eligible woman on our initial household entry, a woman with a recent birth was chosen. A total survey sample size of 90 women across the three communities participated in the survey arm of the study. All interviews were conducted in convenient locations at the community level suin the native Gurene language. After field piloting, a research assistant assisted in the translation of the questionnaire from English to the local language for final administration during data collection. Each questionnaire took an average of 45 minutes to administer.

On the qualitative side of the study, IDIs were conducted concurrently during the survey stage with 15 purposively identified eligible participants across the three communities ( 5 from each community level). In addition, two FGDs were held with a first group $(n=10)$ from both Boko and Namoo communities. The last FGD was conducted in Amanga community with 7 eligible participants. Both FGDs and IDIs were conducted in Amanga community because the community was far away from the main Namoo sub-district health centre. A total of 15 and 17 participants took part in IDIs and FGDs respectively (see table 1). Health staff at two facilities assisted in identifying eligible women for IDIs during the survey administration across the three communities. IDIs for Namoo and Boko respondents took place in Namoo health centre whiles that of Amanga took place at the community level. It was important to validate the views individual responses as this may not reflect majority women views from their community settings. For FGDs, a participant register was opened in Namoo and Boko facility centre with the help of health staff to recruit interested and eligible respondents. To ensure the inclusion of participants from Amanga community since they had no health facility, eligible women from that community were purposively included in each of the other two facility registers. Once an individual expressed interest in participating, health staff provided them information on the study aim and objectives. We allowed a good amount of time for all participants to reflect on their willingness to participate. Those who finally decided to take part in the study were invited to a meeting to discuss their participation and rules of engagement for the study. Respondent's privacy and confidentiality was ensured at every stage of recruitment and data collection. Focus group meetings were conducted in one day in two separate venues in Namoo health centres and Amanga community. Each FGD lasted for an average of one and half hour. Interview guides for FGDs and IDIs were developed based on existing literature and modelled based on SHARE research consortium WASH and clean toolkit developed as part of the Water supply and sanitation collaborative council study. Both in-depth and focus group interview guides allowed some amount of flexibility for participants to ask questions and vice-versa. The specific issues explored were categorized under four themes; perceptions of delivery room conditions, opinions on environmental and safety issues at delivery room, future delivery intentions at the facility level and experiences on informal payments to access maternity care. Daily administered questionnaires were validated to ensure all items were completed. Two research assistants who assisted in data collection cross validated each filled out questionnaire. Seven incomplete questionnaires detected were reviewed and follow up visits made to respondents for further clarification and completion of data.

\section{Data analysis and ethical consideration}

On quantitative data, all validated questionnaires were entered into SPSS version 22 and data cleaned to avoid missing or error entries. Descriptive analysis was applied where individual, household, and other explanatory variables were explored. Individual variables such as women age, marital status, settlement type, educational and employment status, and parity levels were included whiles household variables assessed presence or absence of social amenities (toilet, electricity and water sources) and household size. Binary logistic regression was applied to statistically test associations between socio- 
demographics and other variables. Odd ratios were generated with $95 \%$ CI to determine statistical significant associations influencing women ever experiences, attitudes concerning informal payments and willingness to pay informally for maternity care. Variables were considered statistically significant at $p<0.05$.

On qualitative data, all audio recorded qualitative interviews were transcribed from the local dialect, Gurene to English. Transcripts were developed under the four themes; perceptions of delivery room conditions, opinions on distance and environmental safety issues, intentions for future delivery and informal payments experiences for maternity care. These themes reflected the structure of the qualitative guide applied in the study. All transcriptions were undertaken by two research assistants, with good knowledge of the local dialect. Transcripts were read through several times theme after theme to ensure only significant quotes and all relevant information provided by respondents were captured. All transcripts were manually analysed under each theme applying content analysis.

Written approval was given by the SHARE research consortium to apply their model questions on WASH and clean toolkit in the study. Written and verbal consent was sought from each participant before recruitment and data collection. With permission from each participant all qualitative interviews were audio-recorded and completed with notes taken from the field. Study ethical approval was obtained from the Ethics and Institutional Review Board of the Navrongo Health Research, Formal written permissions were also obtained from the Regional and Bongo District Health Directorates of the Upper East region.

\section{RESULTS}

\section{Quantitative findings}

Socio-demographics of survey respondents: Descriptive statistics of respondents are presented in Table 1. Table 2 presents results of logistic regression to establish factors influencing women ever experiences, attitudes and willingness to pay informally for maternity care. Majority of survey respondents were married (80\%), and unemployed $(55.6 \%)$ with a mean age of 24.4 (SD -4.9). More than half of survey respondents lacked basic social amenities such as water, household toilets and electricity in a mean household of 8 . With an average parity of 3 children, women attested adequate treatment received with more assisted delivery from midwives. Additionally, women opinions on good hygienic hand washing practices by health staffs who conduct deliveries were low. Only $30 \%$ of women were sure of hand washing with soap by health staff prior to conducting deliveries. A relatively high number of survey respondents were not sure $(58.9 \%)$ or asserted hand washing did not take place $(11.1 \%)$ on such occasions. More than half $(62.2 \%)$ of participants confirmed health staff wearing gloves whiles conducting deliveries. An estimated $76.7 \%$ have never paid informally for healthcare. All respondents however have a highly positive $(72.2 \%)$ attitude towards informal payment habits. Majority $(84.4 \%)$ admitted their willingness to pay informally for healthcare with gift payments $(83.3 \%)$ constituting the largest willingness to pay option. On informal payments and amounts if respondents were to pay informally in cash, only $16.7 \%$ are willing to pay between 1-60 Ghana cedi. Access and gratitude are major reasons influencing informal payments among respondents.

\section{Ever experiences for informal payments}

Women who perceive informal payments (cash/gift) as a form of gratitude were five times more willing to pay informally at a health facility $(\mathrm{aOR}=5.5$, 95\% CI 0.903 33.431). Women also asserted informal payments are inevitable $(\mathrm{aOR}=3.263$, 95\% CI 0.5248.651). Gift items was the most desirable payment option ( $\mathrm{aOR}=2.9,95 \%$ CI 0.993-8.673) for accessing and utilizing maternity care compared to cash payments (Table 2).

Individual household characteristics measured by the absence of electricity showed positive association with women offering gift payments to health staff as gratitude for health care services received. Women gifts/cash payments were not perceived as an act of corruption. Informal demands by health staff during service delivery was not positive with women ever experiences with informal payments.

\section{Women attitudes towards informal payments}

Although majority of women had a positive attitude (72.2\%) towards paying informally from table 1 , these attitudes were seen not to influence payments or nonpayments informally. Never educated women ( $\mathrm{p}=0.044$, 95\% CI 1.038-15.040) were three times more likely to show positive attitudes towards informal payments (see Table 2). Women driven perceptions of informal payments as inevitable influenced attitudes $(\mathrm{aOR}=2.437$, 95\% CI 0.225-26.418) towards informal payments. Informal payments initiated by women, husband and other relatives had a twice greater effect $(\mathrm{aOR}=2.213$, $95 \%$ CI 0.883-5.552) on women attitudes towards informal payments compared with informal demands by health staff. Women attitudes towards informal payments were not influenced by settlement type, category of informal gift payment, women ever experience with informal payments and their employment status. Knowledge of where to take complaint for informal payment demands did not influence women attitudes towards informal payments.

\section{Willingness to pay informally}

Women who ever paid informally and those with lower parity levels are more willing to pay informally in accessing maternity care $(\mathrm{aOR}=11.3$, 95\%CI. 0.637- 
200.428, aOR $=11.295 \%$ C.I 1.477-85.293 respectively). Unemployed women were more likely to be willing to pay informally $(\mathrm{aOR}=0.088,95 \%$ CI $0.010-0.773)$ whiles never educated women were three times more likely to be willing to pay informally ( $\mathrm{aOR}=3.080,95 \%$ CI 0.385-24.669). Women lack of knowledge on where to take complaints if demands by health staff were made for informal payments were four times more willing to pay. Women expression of gratitude (aOR $=1.746,95 \% \mathrm{CI}$ 0.186-6.469), and inevitability $(\mathrm{aOR}=4.271,95 \% \mathrm{CI}$ 0.056-326.999) within the health care system for informal payments also influenced their willingness to pay informally in the future. Health staffs demand for payments from women has no association with women intended willingness to pay. Socio-demographic factors such as age, unemployed, settlement type and household conditions (absence of electricity, toilet and water source) had no associations with women willing future intentions for paying informally (Table 3 ).

\section{Qualitative findings}

\section{Women perspectives on facility delivery conditions}

Discussants for IDIs and FGDs showed close consensus and associations on facility delivery conditions, opinions on travel distance and associated safety issues and women intentions for future use. Relatively, a higher number of women during in-depth interviews and focus group discussions asserted that delivery room conditions were clean (table 4 IDI R1 and FGD R5).

However, close to half of interview discussants indicated their experience of poor delivery conditions, with experiences of structural deficits in doors and windows, wet floors, and poor delivery bed conditions prior to delivery. A higher number of IDI discussants indicated they visited the facility prepared, not depending on available facility materials to assist their delivery (table 4 IDI R6).

Women attested to health staff wearing gloves prior to assisting them deliver. However, hand washing by health staff before conducting delivery was observed to be low as more women could not recollect observing these during their last birth.

Additionally, women opinions on distance and environmental safety during delivery largely reflected their views on long travel distance and privacy. Two thirds of all IDI and FGD participants admitted travel time for delivery was a major challenge impacting their decisions to seek facility delivery. In most discussants view, expectant women in far distant communities find it difficult, even given the available transport (Motor tricycles) since it's not comfortable in the midst of poor and undeveloped road networks.

Table 1: Theme categories for qualitative interviews and quantitative data.

\begin{tabular}{|c|c|c|c|}
\hline Participant category (\#) & Method of data collection & Key themes covered & $\begin{array}{l}\text { Sample } \\
\text { number (n) }\end{array}$ \\
\hline \multicolumn{4}{|l|}{ Facility level } \\
\hline $\begin{array}{l}\text { Facility individual level } \\
\text { interactions with; } \\
\text { pregnant women ( } 3 \text { ) } \\
\text { and women with recent } \\
\text { births (12) }\end{array}$ & $\begin{array}{l}\text { IDIs held at three facility/community } \\
\text { level environments that guaranteed } \\
\text { privacy. }\end{array}$ & $\begin{array}{l}\text { Women perceptions of delivery room } \\
\text { conditions. } \\
\text { Opinions on distance, environment and } \\
\text { safety issues at delivery room. } \\
\text { Intentions to undertake future delivery } \\
\text { at facility. }\end{array}$ & 15 \\
\hline $\begin{array}{l}\text { Facility group } \\
\text { discussions with; } \\
\text { group 1, 1 pregnant } \\
\text { woman and women } \\
\text { with recent births (9) } \\
\text { group 2, pregnant } \\
\text { women (2) and women } \\
\text { with recent births (5) }\end{array}$ & $\begin{array}{l}\text { FGDs held at two proximate health } \\
\text { facilities that allowed all IDIs } \\
\text { participants to participate. Two } \\
\text { additional participants included. }\end{array}$ & $\begin{array}{l}\text { Women perceptions of delivery room } \\
\text { conditions. } \\
\text { Opinions on distance, environment and } \\
\text { safety issues at delivery room. } \\
\text { Intentions to undertake future delivery } \\
\text { at facility. } \\
\text { Informal payments experiences, } \\
\text { perceptions and willingness to pay } \\
\text { informally. }\end{array}$ & 17 \\
\hline \multicolumn{4}{|l|}{ Community level } \\
\hline Pregnant women (36) & Semi-structured interviewer & $\begin{array}{l}\text { Women ever experiences of informal } \\
\text { payments }\end{array}$ & 90 \\
\hline $\begin{array}{l}\text { Women with recent } \\
\text { births (54) }\end{array}$ & $\begin{array}{l}\text { Questionnaire administered at } \\
\text { individual household level. }\end{array}$ & $\begin{array}{l}\text { Perceptions on informal payments } \\
\text { Women willingness to pay informally } \\
\text { views on maternity care }\end{array}$ & \\
\hline Total study participants & & & 122 \\
\hline
\end{tabular}


Table 2: Descriptive statistics of sample respondents surveyed.

\begin{tabular}{|c|c|c|c|c|c|}
\hline Variable & Variable description & $\begin{array}{l}\text { Frequency }(\%) \\
\mathrm{N}=90\end{array}$ & Mean & SD & Variance \\
\hline Age & $18-35$ & - & 24.4778 & 4.95197 & 24.522 \\
\hline Marital status & $\begin{array}{l}\text { 1-not married } \\
\text { 2-married }\end{array}$ & $\begin{array}{l}18(20.0) \\
72(80.0)\end{array}$ & 1.8000 & 0.40224 & 0.162 \\
\hline Settlement type & $\begin{array}{l}\text { 1-village/small town } \\
\text { 2-Town }\end{array}$ & $\begin{array}{l}52(57.8) \\
38(42.2)\end{array}$ & 1.4222 & 0.49668 & 0.247 \\
\hline Educational status & $\begin{array}{l}\text { 1-never educated } \\
2 \text {-ever educated }\end{array}$ & $\begin{array}{l}57(63.3) \\
33(36.7)\end{array}$ & 1.3667 & 0.48459 & 0.235 \\
\hline Employment status & $\begin{array}{l}\text { 1-unemployed } \\
\text { 2-employed }\end{array}$ & $\begin{array}{l}50(55.6) \\
40(44.4)\end{array}$ & 1.4444 & 0.49969 & 0.250 \\
\hline Parity levels & $1-7$ & - & 2.6444 & 1.28353 & 1.647 \\
\hline Have $\mathrm{HH}$ toilet & $\begin{array}{l}1 \text {-no } \\
2 \text {-yes }\end{array}$ & $\begin{array}{l}69(76.7) \\
21(23.3)\end{array}$ & 1.2333 & 0.42532 & 0.181 \\
\hline Main water source & $\begin{array}{l}\text { 1-well/borehole } \\
\text { 2-stand tap/pipe water }\end{array}$ & $\begin{array}{l}50(55.6) \\
40(44.4)\end{array}$ & 1.4444 & 0.49969 & 0.250 \\
\hline $\begin{array}{l}\text { Electricity present } \\
\text { at } \mathrm{HH}\end{array}$ & $\begin{array}{l}1 \text { 1-no } \\
2 \text {-yes }\end{array}$ & $\begin{array}{l}70(77.8) \\
20(22.2)\end{array}$ & 1.2222 & 0.41807 & 0.175 \\
\hline $\begin{array}{l}\text { Persons living in } \\
\mathrm{HH}\end{array}$ & $1-25$ & - & 7.6556 & 4.85558 & 23.577 \\
\hline $\begin{array}{l}\text { Health staff washed } \\
\text { hands prior to } \\
\text { delivery }\end{array}$ & $\begin{array}{l}\text { 1-no } \\
2 \text {-not sure } \\
3 \text {-yes }\end{array}$ & $\begin{array}{l}10(11.1) \\
53(58.9) \\
27(30.0)\end{array}$ & 2.1889 & 0.61616 & 0.380 \\
\hline $\begin{array}{l}\text { Health staff wore } \\
\text { gloves prior to } \\
\text { delivery }\end{array}$ & $\begin{array}{l}\text { 1-no } \\
2 \text {-not sure } \\
3 \text {-yes }\end{array}$ & $\begin{array}{l}12(13.3) \\
22(24.4) \\
56(62.2)\end{array}$ & 2.4889 & 0.72274 & 0.522 \\
\hline $\begin{array}{l}\text { Ever demand for } \\
\text { informal payments }\end{array}$ & $\begin{array}{l}\text { 1-no } \\
2 \text {-yes }\end{array}$ & $\begin{array}{l}76(84.4) \\
14(15.6)\end{array}$ & 1.1556 & 0.36446 & 0.133 \\
\hline $\begin{array}{l}\text { Ever paid } \\
\text { informally for } \\
\text { maternity care }\end{array}$ & $\begin{array}{l}\text { 1-no } \\
2 \text {-yes }\end{array}$ & $\begin{array}{l}69(76.7) \\
21(23.3)\end{array}$ & 1.2333 & 0.42532 & 0.181 \\
\hline $\begin{array}{l}\text { Informal payment } \\
\text { type }\end{array}$ & $\begin{array}{l}\text { 1-gifts } \\
\text { 2-money }\end{array}$ & $\begin{array}{l}75(83.3) \\
15(16.7)\end{array}$ & 1.1667 & 0.37477 & 0.140 \\
\hline $\begin{array}{l}\text { Informal payments } \\
\text { initiated by }\end{array}$ & $\begin{array}{l}\text { 1-health staff } \\
\text { 2-self } \\
\text { 3-husbad/other }\end{array}$ & $\begin{array}{l}12(13.3) \\
46(51.1) \\
32(35.6)\end{array}$ & 2.2222 & 0.66667 & 0.444 \\
\hline $\begin{array}{l}\text { Attitude towards } \\
\text { informal payments }\end{array}$ & $\begin{array}{l}\text { 1-negative } \\
\text { 2-positive }\end{array}$ & $\begin{array}{l}25(27.8) \\
65(72.2)\end{array}$ & 1.7222 & 0.45041 & 0.203 \\
\hline $\begin{array}{l}\text { Willing to pay } \\
\text { informally to access } \\
\text { care }\end{array}$ & $\begin{array}{l}1 \text {-no } \\
2 \text {-yes }\end{array}$ & $\begin{array}{l}14(15.5) \\
76(84.4)\end{array}$ & 1.8444 & 0.36446 & 0.133 \\
\hline $\begin{array}{l}\text { Motivation for } \\
\text { willing to pay }\end{array}$ & $\begin{array}{l}\text { 1-quality and gratitude } \\
\text { 2-access and gratitude } \\
3 \text {-access,qualityand } \\
\text { gratitude } \\
\text { 4-gratitude only }\end{array}$ & $\begin{array}{l}21(23.3) \\
35(38.9) \\
25(27.8) \\
9(10)\end{array}$ & 2.2444 & 0.92786 & 0.861 \\
\hline $\begin{array}{l}\text { Amount willing to } \\
\text { pay informally }\end{array}$ & $\begin{array}{l}\text { 1-From 1-20 cedi } \\
\text { 2-From21-40 cedi } \\
\text { 3-From41-60 cedi } \\
\text { 4-From61+ cedi }\end{array}$ & $\begin{array}{l}87(96.7) \\
1(1.1) \\
2(2.2) \\
0\end{array}$ & 3.4778 & 9.40383 & 88.432 \\
\hline
\end{tabular}

CHO- Community health officer, HH-household, cedi-currency exchange for republic of Ghana. 
Table 3: Binary logistic regression of women experiences, attitudes and willingness to pay informally for maternity care.

\begin{tabular}{|c|c|c|c|}
\hline Socio/explanatory variables & $\begin{array}{l}\text { Ever experience } \\
\text { informal payments } \\
\text { aOR ( } 95 \% \text { CI) }\end{array}$ & $\begin{array}{l}\text { Attitudes on informal } \\
\text { payments } \\
\text { aOR ( } 95 \% \text { CI })\end{array}$ & $\begin{array}{l}\text { Willingness to pay } \\
\text { informally for access } \\
\text { aOR ( } 95 \% \text { CI) }\end{array}$ \\
\hline Age & $1.084(0.867-1.355)$ & $1.089(0.900-1.317)$ & $0.597(0.388-0.919) * *$ \\
\hline \multicolumn{4}{|l|}{ Marital status } \\
\hline Not married & $1.325(0.290-6.057)$ & $2.793(0.579-13.475)$ & $1.697(0.165-17.475)$ \\
\hline \multicolumn{4}{|l|}{ Married (ref) } \\
\hline \multicolumn{4}{|l|}{ Educational status } \\
\hline Never educated & $0.825(0.183-3.710)$ & $3.952(1.038-15.040) *$ & $3.080(0.385-24.669) *$ \\
\hline \multicolumn{4}{|l|}{ Ever educated (ref) } \\
\hline \multicolumn{4}{|l|}{ Eemployment status } \\
\hline Unemployed & $0.930(0.234-3.687)$ & $1.497(0.452-4.958)$ & $0.088(0.010-0.773) * *$ \\
\hline \multicolumn{4}{|l|}{ Employed (ref) } \\
\hline Woman parity level & $0.620(0.246-1.563)$ & $0.770(0.374-1.584)$ & $11.223(1.477-85.293) * *$ \\
\hline \multicolumn{4}{|l|}{ Settlement type } \\
\hline Village & $0.640(0.161-2.544)$ & $0.556(0.146-2.121)$ & $0.221(0.023-2.136)$ \\
\hline \multicolumn{4}{|l|}{ Town (ref) } \\
\hline \multicolumn{4}{|l|}{ Household toilet present } \\
\hline No & $0.351(0.072-1.713)$ & $1.001(0.217-4.621)$ & $0.636(0.058-7.015)$ \\
\hline \multicolumn{4}{|l|}{ Yes (ref) } \\
\hline \multicolumn{4}{|l|}{ Household water source } \\
\hline Well/borehole & $1.260(0.292-5.442)$ & $1.575(0.481-5.158)$ & $0.404(0.053-3.079)$ \\
\hline \multicolumn{4}{|l|}{ Stand pipe (ref) } \\
\hline \multicolumn{4}{|l|}{ Electricity present in house } \\
\hline No & $2.025(0.327-12.547)$ & $4.163(0.836-20.721)$ & $0.417(0.024-7.194)$ \\
\hline \multicolumn{4}{|l|}{ Yes (ref) } \\
\hline Household size & $1.059(0.917-1.222)$ & $1.017(0.895-1.157)$ & $1.090(0.863-1.376)$ \\
\hline \multicolumn{4}{|l|}{ Asked to pay informally } \\
\hline No & $0.553(0.091-3.372)$ & $1.019(0.162-6.399)$ & $0.190(0.007-5.410)$ \\
\hline \multicolumn{4}{|l|}{ Yes (ref) } \\
\hline \multicolumn{4}{|c|}{ Know where to take informal payment complaint to } \\
\hline No & $0.303(0.071-1.286)$ & $0.895(0.229-3.492)$ & $3.694(0.528-25.845)$ \\
\hline \multicolumn{4}{|l|}{ Yes(ref) } \\
\hline \multicolumn{4}{|l|}{ Willingness to pay informally } \\
\hline Yes & $0.216(0.021-2.254)$ & $0.559(0.109-2.854)$ & - \\
\hline \multicolumn{4}{|l|}{ No (ref) } \\
\hline Ever experience informal payments & - & $0.643(0.140-2.953)$ & $11.300(0.637-200.428)$ \\
\hline Informal cash or gift is corruption & $0.566(0.117-2.729)$ & $0.738(0.156-3.485)$ & $0.324(0.023-4.564)$ \\
\hline Informal cash or gift is gratitude & $5.494(0.903-33.431) *$ & $0.476(0.113-2.014)$ & $1.746(0.186-6.469) *$ \\
\hline Informal payments are inevitable & $3.263(0.5248 .651) *$ & $2.437(0.225-26.418) * *$ & $4.271(0.056-326.999) *$ \\
\hline Timing for informal payment & $1.211(0.359-4.081)$ & $1.984(0.768-5.122)$ & $3.264(0.579-18.395)$ \\
\hline Who Initiates informal payment & $0.741(0.249-2.206)$ & $2.213(0.883-5.552) * *$ & $0.889(0.162-4.868)$ \\
\hline Pay informally how much (cash) & $1.091(1.014-1.173) *$ & $1.029(0.935-1.133)$ & $1.307(0.755-2.263)$ \\
\hline $\begin{array}{l}\text { What items will constitute your } \\
\text { gifts towards informal payments }\end{array}$ & $2.934(0.993-8.673) *$ & $0.387(0.155-0.967) *$ & $0.522(0.141-1.928)$ \\
\hline $\begin{array}{l}\text { What is your motivation behind } \\
\text { paying informally }\end{array}$ & $1.075(0.482-2.398)$ & $1.562(0.750-3.250)$ & $0.388(0.118-1.276)$ \\
\hline $\begin{array}{l}\text { What is your attitude towards } \\
\text { informal payments }\end{array}$ & $1.714(0.395-7.437)$ & - & $0.332(0.050-2.211)$ \\
\hline -2LL Ratio & $69.970^{\mathrm{a}}$ & $80.620^{\mathrm{a}}$ & $45.199^{\mathrm{a}}$ \\
\hline Cox and Snell R square & 0.266 & 0.249 & 0.304 \\
\hline Nagelkerke R square & 0.401 & 0.359 & 0.525 \\
\hline
\end{tabular}

(ref)-indicates reference categories, $\mathrm{P}<0.05 \square, \mathrm{P}<0.0$. 
Table 4: Significant quotes from IDIs and FGDs for facility delivery and informal payments experiences.

\begin{tabular}{|c|c|c|c|c|}
\hline $\begin{array}{l}\text { Interview } \\
\text { category }\end{array}$ & \multicolumn{3}{|c|}{ IDIs and FGDs for facility Delivery Conditions $(\mathbf{a} / \mathbf{b}) *$} & $\begin{array}{l}\text { FGDs on Informal } \\
\text { payments }(\mathrm{a} / \mathrm{b}) *\end{array}$ \\
\hline Location & $\begin{array}{l}\text { Perceptions of delivery } \\
\text { room conditions }\end{array}$ & $\begin{array}{l}\text { Opinions on distance, } \\
\text { environment and safety } \\
\text { issues at delivery room }\end{array}$ & $\begin{array}{l}\text { Intentions to undertake } \\
\text { any future deliveries } \\
\text { within the facility }\end{array}$ & $\begin{array}{l}\text { Informal payments } \\
\text { expressions }\end{array}$ \\
\hline $\begin{array}{l}\text { Namoo, } \\
\text { Boko and } \\
\text { Amanga } \\
\text { participants }\end{array}$ & $\begin{array}{l}\text { "I think the room was clean. } \\
\text { The bed cover was also good } \\
\text { and the environment. I don't } \\
\text { know others think but to me i1 } \\
\text { was ok" (9/15),IDI } \mathbf{R}^{\mathbf{1}} \\
\text { "When I arrived, there was } \\
\text { blood stains on bed and } \\
\text { floor, but the nurse asked } \\
\text { and it was cleaned before I } \\
\text { was lied on delivery bed" } \\
\text { (6/15) IDI } \mathbf{R}^{\mathbf{2}} \\
\text { "The nurse will inspect the } \\
\text { delivery items your brought } \\
\text { to make sure you have what } \\
\text { they expect before they } \\
\text { start anything" (3/15) IDI } \\
\mathbf{R}^{\mathbf{3}} \\
\text { "I didn't bother myself } \\
\text { about looking at the facility } \\
\text { conditions, all I wanted was } \\
\text { to give birth to my baby } \\
\text { since I was sure it would } \\
\text { come safe" (6/15) IDI } \mathbf{R}^{\mathbf{4}} \\
\text { "Standing by nurses was } \\
\text { shouting at me when I was } \\
\text { in pain, this was very } \\
\text { irritating but I had no } \\
\text { option. (6/15) FGD } \mathbf{R}^{\mathbf{1}} \\
\text { "I prepared my cloths and } \\
\text { good rags ahead of time. So } \\
\text { when I was going, I knew I } \\
\text { will use clean clothes to } \\
\text { spread on bed and delivery } \\
\text { and the once for my baby" } \\
\text { (7/15) IDI } \mathbf{R}^{\mathbf{5}} \\
\text { "I didn't want to depend on } \\
\text { bed sheets and other things } \\
\text { provided at the facility } \\
\text { because they may be dirty } \\
\text { because of my previous } \\
\text { first time experience" } \\
\mathbf{( 4 / 1 5 ) ~ I D I ~} \mathbf{R}^{\mathbf{6}} \\
\text { "When I was delivering my } \\
\text { third baby, there was water } \\
\text { on floor and window weak } \\
\text { with so much cold" } \\
\text { (5/15)DI } \mathbf{R}^{7}\end{array}$ & $\begin{array}{l}\text { "The delivery room is } \\
\text { small with the nurse's } \\
\text { things. Nobody can stay } \\
\text { with you inside when you } \\
\text { need support from a } \\
\text { relation. I think that is a } \\
\text { problem" (9/15) IDI } \mathbf{R}^{\mathbf{8}} \\
\text { " the nurse wore gloves } \\
\text { when they delivered my } \\
\text { baby" (12/15) FGD } \mathbf{R}^{\mathbf{2}} \\
\text { "sometimes when you get } \\
\text { there and the baby is } \\
\text { coming, they just put you } \\
\text { on bed and deliver you } \\
\text { without washing hands" } \\
\mathbf{( 4 / 1 5 ) ~ F G D ~} \mathbf{R}^{\mathbf{3}} \\
\text { "Privacy was poor for me } \\
\text { since the delivery door } \\
\text { was in poor state" (5/15) } \\
\text { IDI } \mathbf{R}^{\mathbf{9}} \\
\text { "I had to cross two rivers } \\
\text { before getting there. I } \\
\text { nearly delivered on my } \\
\text { way to facility" (10/15) } \\
\text { IDI } \mathbf{R}^{\mathbf{1 0}} \\
\text { "The "motor king" cannot } \\
\text { reach here during the } \\
\text { raining season, we suffer } \\
\text { to get to the delivery } \\
\text { centre" (12/15) FGD } \mathbf{R}^{\mathbf{4}} \\
\text { "The delivery room is } \\
\text { inside the clinic where } \\
\text { many people come for } \\
\text { services, its often noisy } \\
\text { and distractions for me } \\
\text { when I as in labour" } \\
\text { (9/15) IDI } \mathbf{R}^{\mathbf{1 1}} \\
\text { "I think getting to the } \\
\text { facility was a challenge } \\
\text { when I was in labour. The } \\
\text { motor king is not } \\
\text { comfortable when labour } \\
\text { contractions starts" } \\
\text { (11/15) IDI } \mathbf{R}^{\mathbf{1 2}} \\
\text { The facility "motor king" } \\
\text { had no fuel and I had to } \\
\text { buy fuel to be taken to the } \\
\text { facility" (12/15) IDI } \mathbf{R}^{\mathbf{1 3}}\end{array}$ & 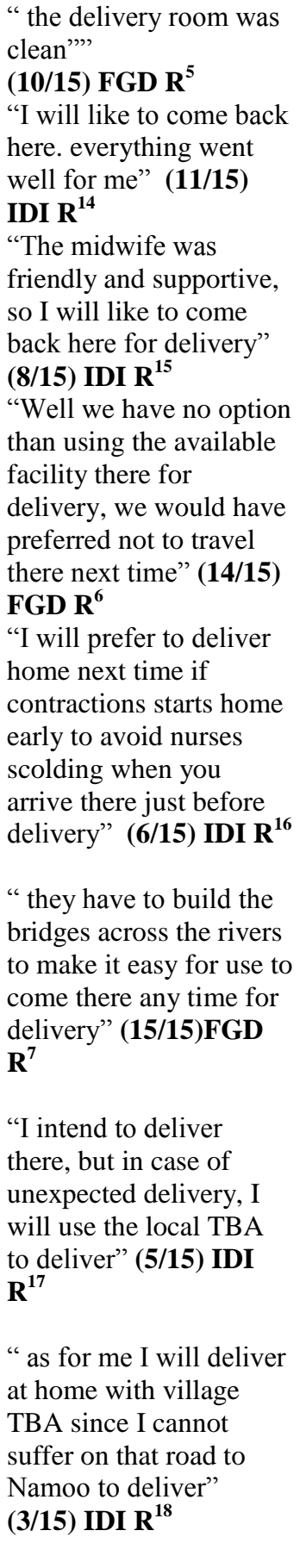 & 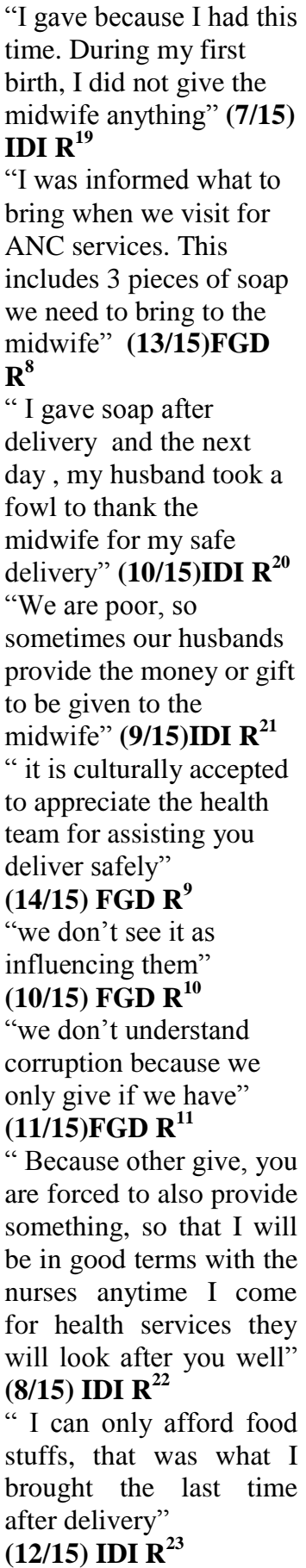 \\
\hline
\end{tabular}

*a/b- a-number of associated view whiles b represents the total population (15) of interview respondents. R1-n-Diffrentiated count of quotes among respondents for IDIs and FGDs.

Women complained about the struggles they go through before reaching a health facility. Even among women who may desire motor tricycle services, meeting financial obligations for the purchase of fuel for the journey is important to access the motor tricycle. Clinic infrastructure was admitted to be poor in guaranteeing privacy and space. Noisy environments emanating from other clinic users and limited space in delivery rooms 
obstructing women desires for family and other relational support during delivery was reported by discussants too (table 4 IDI R8,11). On women's intentions to seek facility delivery during their next births, a clear consensus was observed where women indicated their willingness to return to the facility because of the adequate services they received during their last visit. Women attributed being adequately cared for based on physical treatments received in the hands of facility staff. Others, however would return because they had no other option since they would not want to give birth at home. Close to half of IDI participants however signposted they may seek home delivery if conditions do not change in their subsequent births (table 4 IDI R16).

\section{Women perspectives of Informal payments}

Informal payments was evidenced to be universally acceptable and culturally normal for most women. Women viewed informal payments as respect for normal social value systems that depicted one's social connection and ties to those you provide gifts and show support to. Undoubtedly, focus group discussants intimated their positive attributes towards informal payments and indicated they were socially acceptable and did not amount to inducement or corruption to influence health staff (table 4 FGD R9-11). Few of the discussants however admitted, their gift payment previously was induced by previous women giving since they did not want to suffer any lukewarm treatment when visiting the facility the next time.

\section{DISCUSSION}

\section{Informal payments for maternity care}

In this study, gift payments were widely accepted as a cultural norm with women more willing to pay informally (gifts) as a sign of gratitude to health staff for services received. Due to low economic status, informal payments involving soap and food stuffs was mostly cited. This phenomenon, described in this study as gift assurance is ruled by the cultural perception a good appreciation to a health staff today would yield in friendly and supportive services upon return. Informal payments are also viewed as inevitable within the community cultural setting and transcends beyond the health care system. These payments are often made by husbands as they handle family financial and assets payment options. Informal payment from women's views in this study was inherent and existed as women identified these periods as opportunities to motivate and show appreciation to health staff after delivery. Women's views and perceptions reflect the comfort and value relationship they attach to developing a friendlier relationship with health facility staff for periods of seeking care. Mrisho et al emphasized this value relationship as critical as their study when it was revealed women would most likely deliver with a health provider to whom they previously have a good standing relation with. ${ }^{31}$
Although Ghana introduced a free delivery care policy to remove financial and other payment constraints that affect women utilisation for delivery care, unchecked gift payments as those evidenced in this study could potentially introduce inequality and service inequity issues for women. This could potentially introduce inequality in health access among economically poor and vulnerable groups who seek care at the facility level. Inequity issues are more likely to impact adversely on women with lower educational status and those lacking knowledge of where to complain appropriately when asked to informally pay. This view supports a study in West Africa which indicated that women with poor living conditions were more likely to be associated with a lower perceived ability to overcome their barriers to health seeking. ${ }^{32}$ Surprisingly, women who have ever paid informally and those with lower parity levels were more willing to pay informally again and did not see these payments as acts of corruption. This is potentially possible because women who payed informally might have received friendlier services before and anticipate obtaining the same level of services from facility staff. Younger women desiring for more births may also see the potential need to stay connected with a midwife or clinic staff in anticipation for support during subsequent deliveries. An individual's socio-economic status from this study did not influence payment outputs and attitudes as women paid based on their cultural assertiveness and individual payment abilities. However, lower educated women were more willing to pay informally in gifts. This reveals catastrophic health expenditures are likely to exist indirectly in environments where informal payments are socially or culturally accepted. Women with fewer children and who ever paid informally were more convinced of the potential benefits of providing informal gift assurance for meeting future health needs. Since payments were socially acceptable, formal complaints systems even if they exist at the facility level will not yield the desired public support to eradicate these informal payments that may threaten equity issues at both the individual and household level. A broad stakeholder consensus in dealing with cultural norms that support informal payments at the facility level is important at understanding strategies to divest its effects on equity and access issues.

\section{Delivery conditions at the facility level}

Evidence in this study suggests widespread awareness among women on proper delivery conditions expected from clinic staff. Women asserted delivery rooms conditions were clean, with good relational support from health staff during delivery. However such views were not unanimous as few assertions of blood stains on beds and wet floors were reported by others. However, women were more positive of their future facility utilisation for delivery, emphasizing their perceive trust of facility delivery conditions despite the few shortfalls and challenges in geographical accessibility encountered routinely. Women's views from this study reflect the 
gradual shift and awareness of rural women of the benefits associated with a skilled attendant at delivery as evinced in other studies. ${ }^{33-35}$ This district finding is consistent with another study in northern Ghana that showed women shift towards facility delivery with a widespread understanding of the need for clean delivery practices to prevent neonatal infection during delivery. ${ }^{9}$

Relatively, fewer women highlighted shouting and neglect as some labour room situational conditions encountered, which affects their positive self-worth when considering health seeking at the facility level. It is also difficult to potentially assess if the friendly and supportive attitudes of staff towards most women were potentially influenced by previous informal payments made. It is critical that, poor salient relations cited by few women in this study could potentially mask existing poor geographical access, and women low socio-economic status to affect women access for improved skilled delivery care. This agrees with a similar study in northern Ghana that revealed women perceive these treatments as inhumane, often requiring recurrent assurance, encouragement and friendliness from health staff to enable them return again for services. ${ }^{20,23}$

Birth preparedness education given at antenatal sessions enabled women visit delivery centres prepared with bed linings and soaps as indicated by nurses prior to delivery. Soap for nurse or midwives was singled out however as the most demanded of the items. Although no causeeffect relationship has been established on the effects of this on delivery conditions perceived by women, evidence from this study shows the negative potential impacts as some women intimated home delivery for them could not be ruled out in such instances if clinic staff continue to insist on soap payments after delivery at the facility. The use of safety gloves and hand washing practices was highly attested by women in this study. This differs from another study in Ghana that revealed use of gloves and hand washing was rarely mentioned as practiced by health staff. ${ }^{9}$

Women's understanding and views of how clinic settings could be more organized to accommodate them are expressed in terms of space and privacy in this study. Women's desire to have available walking spaces to accommodate family relations to provide comfort and reassurance during labour was a dominant recurrent expression by the respondents. Women's ascription for privacy was more associated with quite clinic environments that enabled them manage pregnancy contractions and delivery room conditions with fewer obstructions from other clinic users.

Travel distance was intimated as integral to women perceptions on delivery care and support for facility utilisation. Poor road networks and unreliable transport conditions resulting in delays was common as already evidenced in many studies. Travel distance in this study had a potential to shift women's choices from facility delivery to home based delivery given a continual decline of poor road infrastructure with time. Additionally, facility distance expressions from this study create direct distance and opportunity cost and other demand side barriers impacting women views for delivery care conditions and utilisation. This evidence is widely documented within a rural context, with the greatest potential to impact on the most vulnerable..$^{31,36-40}$

The study has limitations. The study only focused on facility delivery conditions, excluding all related clinical delivery conditions at the facility level. This study excluded home conducted deliveries which has the potential to have greater risk of delivery infections due to poor delivery conditions. This supposes that, the views and expressions of women at the facility level in this study cannot be assumed to have captured all women's views and expressions for improving maternity care entirely. They however provide a snapshot of salient drivers of maternity care needs at the facility level that needs to be improved at the system level for greater skilled delivery utilisation.

Delivery conditions and informal payments at clinic settings have always been sensitive to investigate. The nature of these sensitivities could have potentially caused subjective bias response of conditions and informal payments. Interviews were conducted with strong ethical and cultural underpinnings to reduce any potential bias and minimize recall bias among respondents. Views and expressions were only based on women perspectives, excluding clinic staff on measures assessed. Perspectives from health staff would have further provided consensus and aberrations on women perspectives for better decision making. Never the less, women consensus measured in this study provides new insights for improving women delivery conditions in a rural context.

\section{Policy considerations}

At the health policy level in Ghana, evidence from this study provide further evidence on frontline health staff and policy makers at the district levels can help improve maternity service delivery in Ghana. And the following three points illustrate policy options that need greater stakeholder consensus building and action to improve women delivery conditions along the continuum of care in Ghana; Firstly, to meet women expectation for clean, friendlier and supportive delivery environments, a higher degree of quality assurance and supervision is needed at facility levels.

Secondly, government social investments in rural areas seeking to improve the standards of basic infrastructure such as roads and facility infrastructure help improve women urgency for delivery at the facility level. There is urgent need for continual increase of professional staff numbers and skills along the continuum of care especially in rural Ghana. Additionally, a policy option may include government policy of extending the CHPS concept across many far to reach areas to enable women avoid indirect associated cost with delivery. Lastly, a national level 
study to unearth the scale of informal payments within the health system in Ghana is needed. This will enable stakeholder support to design and implement mechanism that reduces informal payment options that threaten and introduce inequity issues at the facility level.

\section{CONCLUSION}

Women desire and perceive access to friendlier and supportive services that meet their expectations for delivery care differ. What constitutes appropriate delivery support and conditions from this study reflects treatment conditions encountered by women at the facility level. To meet expected friendlier and supportive environments, other women may use informal payments as a gift assurance strategy. Stakeholder consensus in dealing with cultural norms that supports informal payments are important as this unchecked cultural norm may bring undue financial burden and catastrophic health expenditure effects for women and their households.

Travel access and distance challenges indicate deplorable road networks in the study district that causes delays in reaching health delivery facilities. Greater rural social investments that improve roads and facility infrastructure can improve women health seeking behaviours urgency at the rural level. At facility levels, a higher degree of quality assurance and supervision is critical to subvert poor delivery conditions and informal payment undertones at the facility level. Whiles this study did not seek to ascertain skilled delivery utilisation, health programming interventions that aim to improve woman perception of good delivery outcome conditions may help target vulnerable groups towards improving skilled delivery care evidenced from this study.

Further evidence of how to build greater consensus on tackling salient undertones of informal payments for healthcare is needed in Ghana. A study that will explore the views of providers, women and policy makers on creating ideal delivery conditions whiles removing illegal payments at the facility level can provide additional shortcomings at the individual, facility and system level for improving maternity care in a rural developing context.

\section{ACKNOWLEDGEMENTS}

Authors acknowledge the regional and district staff that supported in facilitating facility access visits and meetings with women. Our thanks to the Share research consortium on sanitation and hygiene for providing initial basis for this study. We are thankful too for those who peer-reviewed the study for publication.

Funding: No funding sources Conflict of interest: None declared

Ethical approval: Ethical approval was provided by the Navrongo Health Research Centre with approval ID NHRCIRB2O2

\section{REFERENCES}

1. Islam M. Making pregnancy safer. Bulletin of the World Health Organisation. 2007;85.

2. Obaid TA. State of world population 2005.

3. Montagu D, Yamey G, Visconti A, Harding A, Yoong J. Where do poor women in developing countries give birth? A multi-country analysis of demographic and health survey data. PLoS One. 2011;6(2):e17155.

4. Mubyazi GM, Bloch P, Magnussen P, Olsen OE, Byskov J, Hansen KS, Bygbjerg IC. Women's experiences and views about costs of seeking malaria chemoprevention and other antenatal services: a qualitative study from two districts in rural Tanzania. Malar J. 2010;9:54.

5. Solarin I, Black V. They told me to come back: women's antenatal care booking experience in inner-city Johannesburg. Matern Child Health J. 2013;17(2):359-67.

6. Ekott M, Ovwigho U, Ehigiegba A, Fajola A, Fakunle B. Perception of pregnant women about antenatal care in a cottage hospital in port harcourt, Nigeria. Journal of community health. 2013;38(2):360-5.

7. Langer A, Nigenda G, Romero M, Rojas G, Kuchaisit C, Al-Osimi M, Orozco E. Conceptual bases and methodology for the evaluation of women's and providers' perception of the quality of antenatal care in the WHO antenatal care randomised controlled trial. Paediatr Perinat Epidemiol. 1998;12(s2):98-115.

8. Engmann C, Walega P, Aborigo RA, Adongo P, Moyer CA, Lavasani L et al. Stillbirths and early neonatal mortality in rural Northern Ghana. Trop Med Int Health. 2012;17(3):272-82.

9. Moyer CA, Aborigo RA, Logonia G, Affah G, Rominski S, Adongo PB, Williams J, Hodgson A, Engmann C. Clean delivery practices in rural northern Ghana: a qualitative study of community and provider knowledge, attitudes, and beliefs. BMC pregnancy and childbirth. 2012;12(1):50.

10. Bhutta ZA, Darmstadt GL, Hasan BS, Haws RA. Community-based interventions for improving perinatal and neonatal health outcomes in developing countries: a review of the evidence. Pediatrics. 2005;115(Supplement 2):519-617.

11. Asamoah BO, Agardh A, Pettersson KO, Östergren PO. Magnitude and trends of inequalities in antenatal care and delivery under skilled care among different socio-demographic groups in Ghana from 1988-2008. BMC pregnancy and childbirth. 2014;14(1):295.

12. Atunah-Jay SJ, Pettingell S, Ohene SA, Michael Oakes J, Borowsky IW. The relationship between antenatal provider type and maternal care in rural Ghana: a cross-sectional study. Trop Med Int Health. 2013;18(6):678-86.

13. Lambon-Quayefio MP, Owoo NS. Examining the influence of antenatal care visits and skilled delivery 
on neonatal deaths in Ghana. Applied health economics and health policy. 2014;12(5):511-22.

14. Addai I. Demographic and sociocultural factors influencing use of maternal health services in Ghana. Afr J Reprod Health. 1998;2(1):73-80.

15. GSS, GHS, Macro I. Ghana Demopgraphic and Health Survey 2008. Accra Ghana. 2009.

16. MOH. Creating wealth through health. Accra, Ghana, 2007.

17. MOH. National Consultative Meeting on the Reduction of maternal mortality in ghana: partnership for action: a synthesis report. Ministry of Health. 2008.

18. Nguyen $H$, Rajkotia $Y$, Wang $H$. The financial protection effect of Ghana national health insurance scheme: evidence from a study in two rural districts. Int J Equity Health. 2011;10(4):9-10.

19. Nyonator F, Kutzin J. Health for some? The effects of user fees in the Volta Region of Ghana. Health policy and planning. 1999;14(4):329-41.

20. Moyer CA, Adongo PB, Aborigo RA, Hodgson A, Engmann CM. They treat you like you are not a human being: maltreatment during labour and delivery in rural northern Ghana. Midwifery. 2014;30(2):262-8.

21. Bruce J. Fundamental elements of the quality of care: a simple framework. Studies in family planning. 1990:61-91.

22. Langer A, Villar J, Romero M, Nigenda G, Piaggio G, Kuchaisit C, Rojas G, Al-Osimi M, Belizán JM, Farnot U. Are women and providers satisfied with antenatal care? Views on a standard and a simplified, evidence-based model of care in four developing countries. BMC women's health. 2002;2(1):7.

23. d'Ambruoso L, Abbey M, Hussein J. Please understand when I cry out in pain: women's accounts of maternity services during labour and delivery in Ghana. BMC public health. 2005;5(1):140.

24. Moyer CA, Adongo PB, Aborigo RA, Hodgson A, Engmann CM, DeVries R. It's up to the woman's people: how social factors influence facility-based delivery in Rural Northern Ghana. Matern Child Health J. 2014;18(1):109-19.

25. Mills S, Williams J, Adjuik M, Hodgson A. Use of Health professionals for delivery following the availability of free obstetric care in Northern Ghana. Matern Child Health J. 2008;12(4):509-18.

26. Gabrysch S, Campbell OM. Still too far to walk: literature review of the determinants of delivery service use. BMC Pregnancy Childbirth. 2009;9(1):34.

27. Belli P, Gotsadze G, Shahriari H. Out-of-pocket and informal payments in health sector: evidence from Georgia. Health Policy. 2004;70(1):109-23.
28. Stepurko T, Pavlova M, Gryga I, Groot W. Empirical studies on informal patient payments for health care services: a systematic and critical review of research methods and instruments. BMC Health Services Research. 2010;10(1):273.

29. Balabanova D, McKee M. Understanding informal payments for health care: the example of Bulgaria. Health Policy. 2002;62(3):243-73.

30. Thompson R, Witter S. Informal payments in transitional economies: implications for health sector reform. Int $\mathbf{J}$ Health Plann Manage. 2000;15(3):169-87.

31. Rockers P, Wilson M, Mbaruku G, Kruk M. Source of antenatal care influences facility delivery in rural Tanzania: a population-based study. Matern Child Health J. 2009;13(6):879-85.

32. Nikiema B, Haddad S, Potvin L. Measuring women's perceived ability to overcome barriers to healthcare seeking in Burkina Faso. BMC public health. 2012;12(1):147.

33. Kruk ME, Rockers PC, Mbaruku G, Paczkowski MM, Galea S. Community and health system factors associated with facility delivery in rural Tanzania: A multilevel analysis. Health Policy. 2010;97(2):20916.

34. Mpembeni RN, Killewo JZ, Leshabari MT, Massawe SN, Jahn A, Mushi D,et al. Use pattern of maternal health services and determinants of skilled care during delivery in Southern Tanzania: implications for achievement of MDG-5 targets. BMC Pregnancy Childbirth. 2007;7(1):29.

35. Otis KE, Brett JA. Barriers to hospital births: why do many Bolivian women give birth at home? Revista Panamericana de Salud Pública. 2008;24(1):46-53.

36. Ensor T, Cooper S. Overcoming barriers to health service access: influencing the demand side. Health policy and planning. 2004;19(2):69-79.

37. Bove RM, Vala-Haynes E, Valeggia CR. Women's health in urban Mali: Social predictors and health itineraries. J Soc Sci Med. 2012;75(8):1392-9.

38. Arthur E. Wealth and antenatal care use: implications for maternal health care utilisation in Ghana. Health economics review. 2012;2(1):1-8.

39. Atinga RA, Baku AA. Determinants of antenatal care quality in Ghana. Int $J$ Soc Econ. 2013;40(10):852-65.

40. Kowalewski M, Mujinja P, Jahn A. Can mothers afford maternal health care costs? user costs of maternity services in rural Tanzania. Afr J Reprod Health/La Revue Africaine de la Santé Reproductive. 2002;6(1):65-73.

Cite this article as: Ayanore MA, Kuganab-lem R, Aryee PA. Beyond our labour pains: women experiences of informal payments and delivery room conditions at birth in the Bongo district of Ghana. Int J Community Med Public Health 2016;3:1695-706. 